\title{
Better Safe Than Sorry: Ethics Review in European Union-Funded Health Research
}

Ruxandra Draghia-Akli, Arnd Hoeveler, Peter Löffler, and Joana Namorado*

ABSTRACT

Public scrutiny and the increasing number of projects addressing later stages of the research and innovation process announced the need for an enhanced attention to be paid to identify and address ethics concerns. Ex-ante ethics review as implemented in EU-funded health research and a proactive ethics management are a genuinely useful exercise and ensures top-quality research, from the lab to the patient.

\section{Ex-ante ethics review in} health research-why?

Health research is increasingly under public scrutiny, and funders and scientists alike are expected to ensure that it is conducted in an ethical manner. The stakes are high: Ethical standards in medical research ensure respect for all human subjects and they protect their health and rights. They also preserve the reputation of and minimize risks to researchers, projects, and funding bodies alike.

This is particularly true for translational health research, which touches on a significant cascade of ethics considerations and rules, such as informed consent, patient enrollment in clinical trials, research on children or vulnerable human patients, use of nonhuman primates, animal testing, research on human

DG Research \& Innovation, European Commission, Brussels, Belgium.

*(Correspondence: joana.namorado@ec .europa.eu) embryos or fetuses, clinical trials in developing countries, and privacy and data protection. Research in cell therapies is fully exposed to these issues given that, although patient cohorts are small, the intervention is specific and ethical issues are complex.

The European Commission uses an exante approach to anticipate and mitigate potential problems as early as possible across the whole range of European Union (EU) funding mechanisms in the domains of health research and innovation-from single researcher grants, small and medium enterprise support, and basic research to collaborative translational studies in large international teams. Built into the planning stage of the research, it works by identifying ethics issues and risks, and by providing guidance for addressing them before the funded activities start. It allows scientifically sound and "good practice" research to proceed unhindered, while, at the same time, providing assurance that EU-funded research is subject to rigorous ethics scrutiny and international ethics standards.

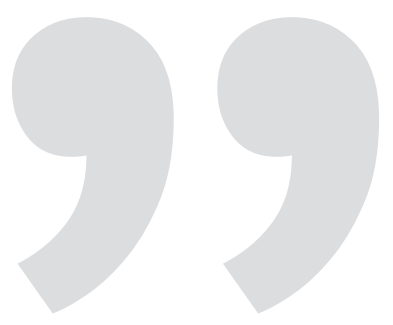

The ethics screening originally developed for the EU's health research program will be in use throughout the commission in all research areas of Horizon 2020.

\section{Efficiency gains through ethics screening}

The principle of reviewing the ethics aspects of applications for EU research funding dates back to the start of the EU's fourth research program some 20 years ago. The need for this step was largely acknowledged, but it came with the realization of the additional scrutiny and effort that it implied. Only the proposals selected for funding undergo ethics review, when flagged by the applicants, by the scientific reviewers, or by scientific officers of the European Commission. Over time, more and more ethics aspects were identified, the ethics environment became more stringent, and a large number of projects required ethics review, with the recommendations of the ethics review to be included in the project work plan.

In 2007, the EU's 7th research framework program FP7 [1] introduced new ethics rules to improve and streamline the process. The number of research proposals received went up dramatically, 
Table 1. FP7 Health Research Projects Undergoing Ethics Screening and Full Ethics Review [2]

\begin{tabular}{|l|c|c|c|c|c|c|}
\hline & 2007 & 2008 & 2009 & 2010 & 2011 & 2012 \\
\hline Ethics screening & 221 & 127 & 132 & 162 & 156 & 189 \\
Full ethics review & 117 & 80 & 56 & 49 & 47 & 58 \\
\hline
\end{tabular}

proportionate to the increase in funding, complexity of projects, and proposals addressing later stages of the research and innovation continuum (clinical trials, advanced therapies, cohort studies, etc.). To get these cutting-edge projects up and running quickly but scrutinize their ethics issues carefully at the same time, the commission built an "ethics screening" phase into the overall evaluation process to check all the successful research proposals, identify ethics concerns, and give applicants the chance to provide relevant documents before an ethics review panel would scrutinize them in depth.

Although the number of proposals to be screened has gone up significantly in recent years, the projects that had to undergo complete ethics review remained fairly constant. For the last FP7 health research funding round in April 2013, 149 proposals had to be screened and 58 were finally referred to ethics review (Table 1) [2].

\section{How does it work in practice?}

The current ethics evaluation of EUfunded health research projects is a welldesigned process, reflecting the particular challenges that each project presents. Projects are often large and complex, involving many partners from different countries and including a wide array of tasks that are carried out under various national legislations. From an ethics point of view, this translates into potential issues and the need to comply with specific national regulatory requirements that exist in addition to the relevant EU rules, such as the European legislation on clinical trials, animal welfare, or data protection. For instance, human stem cell research policy and regulatory frameworks vary largely between different European countries [3], reflecting also public acceptance in the 28 EU member states with different traditions and cultural backgrounds.

Ethics review is conducted centrally. The reviewers are independent external experts carefully selected by the commission for their particular knowledge and experience in the field. They travel to Brussels to read and discuss the project proposals in person, to be able to immediately exchange their views and agree on common conclusions. The commission also makes sure that the evaluators represent the right mix of competences, to do justice to various aspects of the research proposals. They include scientists, ethicists, lawyers, public regulators specialized in health, and representatives of industry and patient groups. Altogether, 25 experts from 12 different EU and other countries ${ }^{1}-14$ men and 11 women-were involved in ethics review during the FP7 program.

As a first step, all research proposals submitted undergo ethics screening. No exceptions to this rule are made, because more than 9 out of 10 proposals raise ethics issues. Data processing and privacy concerns are among the most frequently found - in about $30 \%$ of the proposalsyet they are difficult to spot by nonspecialists as most of us ignore the complexity and possibilities of modern

${ }^{1}$ Belgium, Switzerland, Germany, Estonia, Spain, Finland, France, Ireland, Lithuania, Portugal, Sweden, United Kingdom, and the United States. data gathering and processing technologies, let alone stay abreast of their rapid evolution. Other relatively frequent ethics issues include dual use, research in humans and in animals, and international cooperation with partner countries [2].

Following independent readings and discussion by the reviewers (in more complex cases up to seven), the proposals are then grouped into three categories:

\section{Proposals with no apparent ethical concerns.}

In this case, the research can be funded and carried out as outlined, without any modifications on ethical grounds.

2. Proposals in which there are ethics issues governed by EU law (data protection, clinical trials, animal welfare, etc.) and that require an approval and/or a positive opinion at the appropriate local or national level.

The commission will take the ethics screening report into account when funding a proposal and, if necessary, it can request changes to the proposed research. The applicant must send copies of the requested ethics approvals, authorizations, and so forth to the commission before the research starts.

3. Proposals that require an in-depth ethics review, due to the nature of the ethical issues raised.

This is primarily research involving severe interventions on humans and children, and research using nonhuman primates, human embryos, or human embryonic stem cells. It may also include considerations such as toxicity, biosafety, 
containment, commercialization and dual use, or data processing and privacy. These proposals are read by at least five reviewers, followed by in-depth discussions of relevant issues. They draw up an ethics review report with very precise requirements to ensure that the research project will be carried out in strict conformity to the applicable standards and guidelines. This report is forwarded to the applicants, and its requirements have to be incorporated into the research project before it can receive a funding contract.

This three-category system has proved to be very efficient. Proposals with few, if any, ethical issues can be speedily handled and move swiftly to the conclusion of the funding contract. Full attention can then be given to those proposals that have significant issues and that were singled out for a more detailed ethics review.

\section{The benefits of getting your ethics framework in order}

The combination of an all-inclusive ethics screening with a more in-depth ethics review of selected proposals demonstrated its efficiency and positive results in the past years. Originally developed for the EU's health research program, it will be in use throughout the commission, in all research areas of the new EU research framework program "Horizon 2020” [4].

\section{What's Next for EU-Funded Health Research? Horizon 2020}

Horizon 2020 is the financial arm of the Innovation Union, a European Union initiative aimed at securing Europe's global competitiveness. Horizon 2020 consists of nearly $€ 80$ billion (101.9 billion USD) of funding available from 2014 to 2020. Further information on ethical research requirements within Horizon 2020 is available at: http://ec .europa.eu/programmes/horizon2020 /en/h2020-section/ethics
The need for an ethics assessment in health research is beyond question. Almost all of the more than 1000 collaborative health research projects funded by the EU during the last 7 years had to pay attention to specific ethics concerns. The ethics screening and review process helped identify and address them systematically. As a result, not a single project had to be suspended or had serious problems because of unsolved ethics issues.

From the researchers' perspective, ethics screening and review require a meticulous follow-up of the reviewers' findings and recommendations. This being said, the reality is that no project can or should avoid having to comprehensively address ethics questions, and therefore must get its ethics framework in order, including dealing with the necessary rules and approvals. The process applied in EUfunded health research is thus an opportunity to build the project on safe ground from the beginning. The EU can carry out an ethics audit at any time during and up to 2 years after the end of a project, and in the case of research involving the use of human embryonic stem cells, there is even a specific figure-namely, 30\% for the total amount of research activities that are to be audited.

Researchers and companies will also see their return on the investment in their ethics framework when moving from the basic research to the regulatory process to obtain a market license for drugs, devices, procedures, and treatments. Documenting each step, and ensuring traceability will save the rush, anxiety, and waste of time at the end of this innovation process.

A good example is the EU-funded NeuroStemCell project, which took the management of ethical aspects beyond pure compliance to address important ethical questions pertaining to its research in a proactive way. The project, which developed stem-cell-based therapies for Parkinson's disease and Huntington's disease toward clinical application, has also worked on the conditions for clinical trials in patients with these diseases to be ethically acceptable, and has published two articles on this body of work $[5,6]$.

This example shows that ethics review in EU health research and proactive ethics management are not only a genuinely useful exercise but also quite simply a matter of ensuring top-quality researchbecause excellent ethics, from the lab to the patient, are a key feature of overall research excellence.

\section{Legal disclaimer}

The views expressed in this article are the sole responsibility of the authors and do not necessarily reflect the views of the European Commission. Neither the European Commission nor any person acting on behalf of the commission is responsible for the use that might be made of the information contained therein.

\section{Author Disclosure}

\section{Statement}

No competing financial interests exist.

\section{REFERENCES}

1. Decision No $1982 / 2006 / \mathrm{EC}$ of the European Parliament and of the Council of 18 December 2006 concerning the Seventh Framework Programme of the European Community for Research, Technological Development and Demonstration Activities (2007-2013). Official Journal of the European Union L 412/1 of 30 December 2006.

2. Gans-Combe C, J Houghton, A Kurtz, AS Carvalho and G Hermeren. (2014). Health Ethics Screening Assessment: Health Ethics Screening Impact Analysis-FP6/FP7. A working group report. Publication expected in November 2014.

3. Campo-Ruiz V, ed. (2013). Human Stem Cell Research and Regenerative Medicine. European Science Foundation, Strasbourg, France.

4. Regulation (EU) No. 1291/2013 of 
the European Parliament and of the Council of 11 December 2013 establishing Horizon 2020-the Framework Programme for Research and Innovation (2014-2020) and repealing Decision No 1982/2006/EC. Official Journal of the
European Union L $347 / 104$ of 20 December 2013.

5. Hug K and G Hermerén. (2012). Which patient groups should be asked to participate in first-in-human trials of stem-cell-based therapies? J Clin Ethics 23:256-271.
6. Hug K and G Hermerén. (2013). Differences between Parkinson's and Huntington's diseases and their role for prioritization of stem cell -based treatments. Curr Mol Med 13:777791. 